\title{
Dilepton signatures of family non-universal $\mathrm{U}(1)^{\prime}$
}

\author{
Alper Hayreter \\ Department of Physics, Izmir Institute of Technology, IZTECH, Izmir, TR 35430, Turkey
}

Received 29 January 2007; received in revised form 23 March 2007; accepted 27 March 2007

Available online 4 April 2007

Editor: M. Cvetič

\begin{abstract}
The supersymmetric models extending the minimal supersymmetric Standard Model (MSSM) by an additional Abelian gauge factor U(1)' in order to solve the $\mu$ problem do generically suffer from anomalies disrupting the gauge coupling unification found in the MSSM. The anomalies are absent if the minimal matter content necessitated by the $\mu$ problem is augmented with exotic matter species having appropriate quantum numbers. Recently, it has been shown that anomaly cancellation can also be accomplished by introducing family non-universal U(1) ${ }^{\prime}$ charges and non-holomorphic soft-breaking terms [D.A. Demir, G.L. Kane, T.T. Wang, Phys. Rev. D 72 (2005) 015012, hep-ph/0503290]. We discuss collider signatures of anomaly-free family non-universal $\mathrm{U}(1)^{\prime}$ model by analyzing dilepton production in future colliders. We find that, both at LHC and NLC, one can establish existence/absence of such a $Z^{\prime}$ boson by simply comparing the number of dilepton production events for electron, muon and tau lepton. The signal is free of the SM background.
\end{abstract}

(c) 2007 Elsevier B.V. All rights reserved.

\section{Introduction}

The Minimal Supersymmetric Standard Model (MSSM), devised to solve the gauge hierarchy problem of the Standard Model of electroweak interactions (SM), suffers from a serious naturalness problem associated with the Dirac mass of Higgsinos in the superpotential. Namely, the dimensionful parameter $\mu$ contained in the superpotential

$\hat{W} \ni \mu \hat{H}_{u} \cdot \hat{H}_{d}$

is nested in the supersymmetric sector of the theory, and its scale is left completely arbitrary as it is not related to the soft supersymmetry-breaking terms [1]. A way out of this problem is to generate $\mu$ parameter dynamically via the vacuum expectation value (VEV) of some SM-singlet chiral superfield. The extension by a non-SM chiral superfield may or may not involve gauge extension. Concerning the former, the most conservative approach is to extend the gauge structure of the MSSM by an extra Abelian group factor $\mathrm{U}(1)^{\prime}$ along with an additional chiral superfield $\hat{S}$ whose scalar component generates an effective $\mu$ parameter upon spontaneous $\mathrm{U}(1)^{\prime}$ breakdown. What this ad-

E-mail address: alperhayreter@iyte.edu.tr. ditional gauge symmetry actually does is to forbid the presence of a bare $\mu$ parameter as in (1) [2-4]. An important property of $\mathrm{U}(1)^{\prime}$ models is that the lightest Higgs boson weighs significantly heavier than $M_{\mathrm{Z}}$ even at tree level with small $\tan \beta$. Hence the existing LEP bounds $[5,6]$ are satisfied with almost no need for large radiative corrections [7-10]. Besides, they offer a rather wide parameter space for facilitating the electroweak baryogenesis [11].

An important problem in $\mathrm{U}(1)^{\prime}$ models concerns the cancellation of anomalies. Indeed, for making the theory anomaly-free the usual approach to $\mathrm{U}(1)^{\prime}$ models is to add several exotics to the spectrum [12]. This naturally happens in $\mathrm{U}(1)^{\prime}$ models following from SUSY GUTs e.g. $E_{6}$ unification. However, this not only causes a significant departure from the minimal structure but also disrupts the gauge coupling unification-one of the fundamental predictions of the MSSM with weak scale soft masses. Therefore, it would be of greatest interest to keep gauge unification with minimal matter content. This has been accomplished in [13] by introducing family non-universal $\mathrm{U}(1)^{\prime}$ charges in a way solving all anomaly conditions, including the gravitational one.

In this work, we will discuss dilepton signatures of $\mathrm{U}(1)^{\prime}$ models with universal as well as non-universal $\mathrm{U}(1)^{\prime}$ charges in a comparative fashion. Our discussion will include both lepton 
(the ILC) and hadron (the LHC) colliders. At the Born level the cross sections are sensitive to $Z^{\prime}$ exchange only. Therefore, our analysis will have examined $Z^{\prime}$ properties via dilepton signal. The collider signatures of various $\mathrm{U}(1)^{\prime}$ models have already been analyzed in the literature [14-16]. In addition, the $\mathrm{U}(1)^{\prime}$ models have also been tested under electroweak precision bounds [17].

\section{The $\mathrm{U}(1)^{\prime}$ model}

In $\mathrm{U}(1)^{\prime}$ models the MSSM gauge group is extended to include an extra Abelian group factor at the weak scale: $\mathrm{SU}(3)_{C} \times$ $\mathrm{SU}(2)_{L} \times \mathrm{U}(1)_{Y} \times \mathrm{U}(1)^{\prime}$ with respective gauge couplings $g_{3}$, $g_{2}, g_{1}$ and $g_{1}^{\prime}$. The particle spectrum of the model is that of the MSSM plus an MSSM gauge singlet $S$ charged under only the $\mathrm{U}(1)^{\prime}$ invariance. We employ a rather general $\mathrm{U}(1)^{\prime}$ charge assignment as tabulated in Table 1.

As shown in [13], this general $U(1)^{\prime}$ charge assignment suffices to solve all anomaly cancellation conditions in a way respecting the gauge invariance of the superpotential. In fact, one finds the solutions [13]

$Q_{Q_{1}}^{\prime}=Q_{Q_{2}}^{\prime}=Q_{Q_{3}}^{\prime}=\frac{1}{9}\left(3 Q_{E_{2}^{c}}^{\prime}+3 Q_{L_{2}}^{\prime}+Q_{S}^{\prime}\right)$,

$Q_{D_{1}^{c}}^{\prime}=Q_{D_{2}^{c}}^{\prime}=Q_{D_{3}^{c}}^{\prime}=\frac{1}{9}\left(6 Q_{E_{2}^{c}}^{\prime}+6 Q_{L_{2}}^{\prime}-Q_{S}^{\prime}\right)$,

$Q_{U_{1}^{c}}^{\prime}=Q_{U_{2}^{c}}^{\prime}=Q_{U_{3}^{c}}^{\prime}=\frac{1}{9}\left(-12 Q_{E_{2}^{c}}^{\prime}-12 Q_{L_{2}}^{\prime}-Q_{S}^{\prime}\right)$,

$Q_{L_{1}}^{\prime}=-2 Q_{E_{2}^{c}}^{\prime}-3 Q_{L_{2}}^{\prime}, \quad Q_{L_{3}}^{\prime}=-Q_{E_{2}^{c}}^{\prime}-Q_{L_{2}}^{\prime}$,

$Q_{E_{1}^{c}}^{\prime}=3 Q_{E_{2}^{c}}^{\prime}+4 Q_{L_{2}}^{\prime}, \quad Q_{E_{3}^{c}}^{\prime}=2 Q_{E_{2}^{c}}^{\prime}+2 Q_{L_{2}}^{\prime}+Q_{S}^{\prime}$,

$Q_{H_{d}}^{\prime}=-Q_{E_{2}^{c}}^{\prime}-Q_{L_{2}}^{\prime}-Q_{S}^{\prime}, \quad Q_{H_{u}}^{\prime}=Q_{E_{2}^{c}}^{\prime}+Q_{L_{2}}^{\prime}$

in terms of the three free charges:

$Q_{L_{2}}^{\prime}=2, \quad Q_{E_{2}^{c}}^{\prime}=-3, \quad Q_{S}^{\prime}=3$,

in the theory, these free charges are normalized with a factor $C_{Z^{\prime}}$ which varies with the normalizing model.

In what follows, we will use this general solution of the charges in analyzing the collider signatures of family nonuniversal $\mathrm{U}(1)^{\prime}$. The theory consists of three gauge bosons: the photon, the $\mathrm{Z}$ boson and the $\mathrm{Z}^{\prime}$ boson. We parameterize couplings of these vector bosons to fermions via the effective Lagrangian [18]:

$\mathcal{L}_{\text {eff }}=\frac{g_{2}}{4 \cos \theta_{W}} \sum_{i} \bar{f}_{i} \gamma^{\mu}\left(v_{V}^{f}-a_{V}^{f} \gamma^{5}\right) f_{i} \mathrm{~V}_{\mu}$,

where $V=\gamma, Z, Z^{\prime}$, and $f_{i}$ stands for any of the quarks or leptons. The $\mathrm{U}(1)^{\prime}$ gauge coupling $g_{1}^{\prime}$ is included in the vector couplings $v_{V}^{f}$ and axial-vector couplings $a_{V}^{f}$ via the relations

$v_{V}^{f}=2 \cos \theta_{W}\left(Q_{f_{L}}^{\prime}-Q_{f_{R}}^{\prime}\right) \frac{g_{1}^{\prime}}{g_{2}}$,

$a_{V}^{f}=2 \cos \theta_{W}\left(Q_{f_{L}}^{\prime}+Q_{f_{R}}^{\prime}\right) \frac{g_{1}^{\prime}}{g_{2}}$,

where $\theta_{W}$ is the Weinberg angle, and $Q_{f_{L}}^{\prime}$ and $Q_{f_{R}}^{\prime}$ are $\mathrm{U}(1)^{\prime}$ charges of left- and right-handed fermions, respectively.
Table 1

The gauge quantum numbers of chiral superfields of $i$ th family

\begin{tabular}{lllcl}
\hline & $\mathrm{SU}(3)_{c}$ & $\mathrm{SU}(2)_{L}$ & $\mathrm{U}(1)_{Y}$ & $\mathrm{U}(1)^{\prime}$ \\
\hline$Q_{i}$ & $\mathbf{3}$ & $\mathbf{2}$ & $1 / 6$ & $Q_{Q_{i}}^{\prime}$ \\
$U_{i}^{c}$ & $\overline{\mathbf{3}}$ & $\mathbf{1}$ & $-2 / 3$ & $Q_{U_{i}^{c}}^{\prime}$ \\
$D_{i}^{c}$ & $\overline{\mathbf{3}}$ & $\mathbf{1}$ & $1 / 3$ & $Q_{D_{i}^{c}}^{\prime}$ \\
$L_{i}$ & $\mathbf{1}$ & $\mathbf{2}$ & $-1 / 2$ & $Q_{L_{i}}^{\prime}$ \\
$E_{i}^{c}$ & $\mathbf{1}$ & $\mathbf{1}$ & 1 & $Q_{E_{i}^{c}}^{\prime}$ \\
$H_{u}$ & $\mathbf{1}$ & $\mathbf{2}$ & $1 / 2$ & $Q_{H_{u}}$ \\
$H_{d}$ & $\mathbf{1}$ & $\mathbf{2}$ & $-1 / 2$ & $Q_{H_{d}}^{\prime}$ \\
$S$ & $\mathbf{1}$ & $\mathbf{1}$ & 0 & $Q_{S}^{\prime}$ \\
\hline
\end{tabular}

In writing (4) we have neglected the mixing between $\mathrm{Z}$ and $\mathrm{Z}^{\prime}$ bosons. This mixing can stem from kinetic mixing or can be induced after electroweak breaking [7,19]. In this work we neglect such mixings in accord with the experimental bounds that $\alpha_{Z-Z^{\prime}}$ cannot exceed a few $10^{-3}$. This smallness of the mixing puts stringent bounds on the ranges of the soft-breaking masses as it was analyzed in detail in [7,13].

\section{Dilepton signatures of $\mathbf{U}(1)^{\prime}$}

In this section we will analyze the family non-universal $\mathrm{U}(1)^{\prime}$ model by considering its signatures for dilepton production at lepton and hadron colliders, separately. We will investigate distinctive signatures of the $\mathrm{U}(1)^{\prime}$ model under concern with respect to a typical family universal $\mathrm{U}(1)^{\prime}$ model which we choose to be the $\mathrm{U}(1)_{\eta}$ model following from E(6) GUT. The requisite vector and axial-vector couplings of photon, $\mathrm{Z}$ and $\mathrm{Z}^{\prime}$ bosons are tabulated in Tables 2 and 3 for family universal and non-universal models, respectively.

In general, the $2 \rightarrow 2$ scattering process

$f \bar{f} \rightarrow \ell^{+} \ell^{-}$,

where $f$ stands for quarks (hadron colliders) or leptons (lepton colliders) and $\ell$ for any of the charged leptons. This process proceeds with $\gamma, \mathrm{Z}$ and $\mathrm{Z}^{\prime}$ exchanges in the $s$-channel when $\ell$ is not identical to $f$, and in both $s$ - and $t$-channels when $f \equiv \ell$. If center of mass energy of the collider is high enough then $Z^{\prime}$ effects can be disentangled from those of $\gamma$ and $\mathrm{Z}$.

After summing over final-state polarizations and averaging over the initial-state ones, the amplitude-squared of (6) takes the form

$$
\begin{aligned}
\left\langle\left|\mathcal{A}\left(f \bar{f} \rightarrow \ell^{+} \ell^{-}\right)\right|^{2}\right\rangle_{\text {polar }}= & F(s ; v, a)\left[(s+t)^{2}+t^{2}\right] \\
& +G(s ; v, a)\left[(s+t)^{2}-t^{2}\right],
\end{aligned}
$$

where $F(s ; v, a)$ and $G(s ; v, a)$ are given by [18]

$F(s ; v, a)=2 \sum_{\alpha, \beta} \frac{\left(v_{\alpha}^{f} v_{\beta}^{f}+a_{\alpha}^{f} a_{\beta}^{f}\right)\left(v_{\alpha}^{l} v_{\beta}^{l}+a_{\alpha}^{l} a_{\beta}^{l}\right)}{\left(s-M_{\alpha}^{2}+i M_{\alpha} \Gamma_{\alpha}\right)\left(s-M_{\beta}^{2}-i M_{\beta} \Gamma_{\beta}\right)}$

and

$G(s ; v, a)=2 \sum_{\alpha, \beta} \frac{\left(v_{\alpha}^{f} a_{\beta}^{f}+v_{\beta}^{f} a_{\alpha}^{f}\right)\left(v_{\alpha}^{l} a_{\beta}^{l}+v_{\beta}^{l} a_{\alpha}^{l}\right)}{\left(s-M_{\alpha}^{2}+i M_{\alpha} \Gamma_{\alpha}\right)\left(s-M_{\beta}^{2}-i M_{\beta} \Gamma_{\beta}\right)}$. 
Table 2

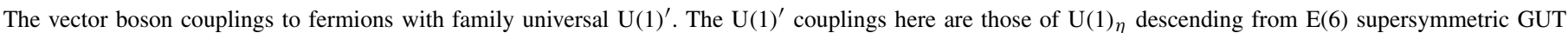
(see [15])

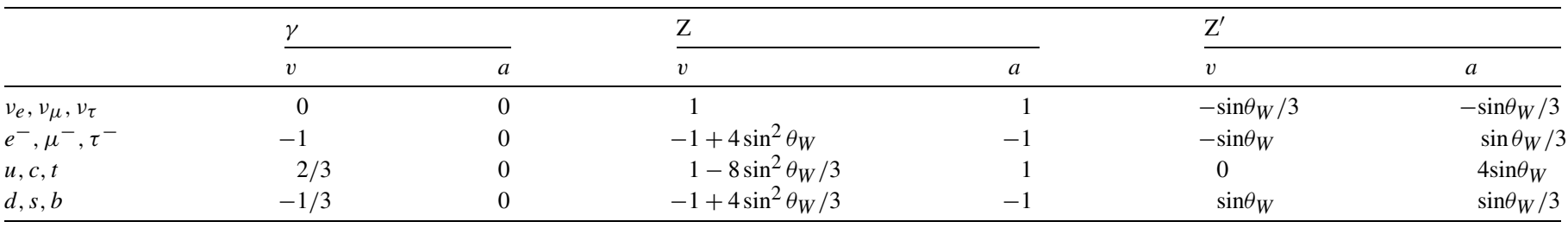

Table 3

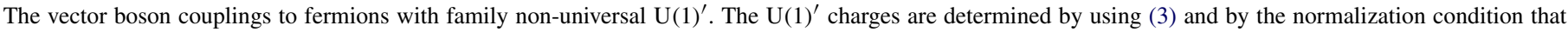
$g_{1}^{\prime 2} \operatorname{Tr}\left[Q^{\prime 2}\right]$ to be equal to the same quantity computed in $\mathrm{U}(1)_{\eta}$ model and the normalization factor $C_{\mathrm{Z}^{\prime}}$ is evaluated as $\sqrt{\frac{5}{52}}$

\begin{tabular}{|c|c|c|c|c|c|c|}
\hline & \multicolumn{2}{|l|}{$\gamma$} & \multicolumn{2}{|l|}{$\mathrm{Z}$} & \multicolumn{2}{|l|}{$\mathrm{Z}^{\prime}$} \\
\hline & $v$ & $a$ & $v$ & $a$ & $v$ & $a$ \\
\hline$v_{e}$ & 0 & 0 & 1 & 1 & $2 \sin \theta_{W} C_{Z^{\prime}}$ & $-2 \sin \theta_{W} C_{Z^{\prime}}$ \\
\hline$v_{\mu}$ & 0 & 0 & 1 & 1 & $10 \sin \theta_{W} C_{Z^{\prime}}$ & $-2 \sin \theta_{W} C_{Z^{\prime}}$ \\
\hline$v_{\tau}$ & 0 & 0 & 1 & 1 & 0 & $4 \sin \theta_{W} C_{Z^{\prime}}$ \\
\hline$e^{-}$ & -1 & 0 & $-1+4 \sin ^{2} \theta_{W}$ & -1 & $2 \sin \theta_{W} C_{Z^{\prime}}$ & $-2 \sin \theta_{W} C_{Z^{\prime}}$ \\
\hline$\mu^{-}$ & -1 & 0 & $-1+4 \sin ^{2} \theta_{W}$ & -1 & $10 \sin \theta_{W} C_{Z^{\prime}}$ & $-2 \sin \theta_{W} C_{Z^{\prime}}$ \\
\hline$\tau^{-}$ & -1 & 0 & $-1+4 \sin ^{2} \theta_{W}$ & -1 & 0 & $4 \sin \theta_{W} C_{Z^{\prime}}$ \\
\hline$u, c, t$ & $2 / 3$ & 0 & $1-8 \sin ^{2} \theta_{W} / 3$ & 1 & $-2 \sin \theta_{W} C_{Z^{\prime}}$ & $2 \sin \theta_{W} C_{Z^{\prime}}$ \\
\hline$d, s, b$ & $-1 / 3$ & 0 & $-1+4 \sin ^{2} \theta_{W} / 3$ & -1 & $2 \sin \theta_{W} C_{Z^{\prime}}$ & $-2 \sin \theta_{W} C_{Z^{\prime}}$ \\
\hline
\end{tabular}

In these expressions $\alpha$ and $\beta$ label intermediate vector bosons i.e. $\gamma, \mathrm{Z}$ and $\mathrm{Z}^{\prime}$. The $\Gamma_{\alpha}$ designates widths of the vector bosons: $\Gamma_{\gamma}=0$ (absolutely stable) and $\Gamma_{\mathrm{Z}}=2.4952 \mathrm{GeV}$. The $\mathrm{Z}^{\prime}$ width $\Gamma_{Z^{\prime}}$ is a model-dependent quantity, and while making numerical estimates in what follows we will take $\Gamma_{\mathrm{Z}^{\prime}}=\Gamma_{\mathrm{Z}}$. Moreover, in accord with the $\mathrm{U}(1)_{\eta}$ model parameter space, we take $g_{1}^{\prime}=g_{1}$.

\subsection{The linear collider signatures}

We first examine $\mathrm{U}(1)^{\prime}$ model at a high-energy linear collider (such as the International Linear Collider (ILC) project under preparation) running at $\sqrt{s}=500 \mathrm{GeV}$. The basic processes we consider are $e^{+} e^{-} \rightarrow \mu^{+} \mu^{-}$and $e^{+} e^{-} \rightarrow \tau^{+} \tau^{-}$where we discard $e^{+} e^{-}$final states simply for avoiding the $t$-channel contributions.

Depicted in Fig. 1 are unpolarized $\mu^{+} \mu^{-}$and $\tau^{+} \tau^{-}$production cross sections

$\sigma\left(e^{+} e^{-} \rightarrow \ell^{+} \ell^{-}\right)=\frac{1}{16 \pi s} \int_{-s}^{0} d t\left\langle\left|\mathcal{A}\left(f \bar{f} \rightarrow \ell^{+} \ell^{-}\right)\right|^{2}\right\rangle_{\text {polar }}$

at a future $e^{+} e^{-}$machine for family universal $\mathrm{U}(1)^{\prime}$ (in the left panel) and family non-universal $\mathrm{U}(1)^{\prime}$ (in the right panel) models. For family universal $\mathrm{U}(1)^{\prime}$ it is seen that $\sigma\left(e^{+} e^{-} \rightarrow\right.$ $\left.\mu^{+} \mu^{-}\right)$and $\sigma\left(e^{+} e^{-} \rightarrow \tau^{+} \tau^{-}\right)$completely overlap. The main reason behind this coincidence is that $\mu$ and $\tau$ leptons do have identical gauge quantum numbers (including those of under the $\mathrm{U}(1)^{\prime}$ gauge symmetry) and their mass difference causes only a tiny deviation at such high energies $[5,6]$. Consequently, from the left panel of Fig. 1 one concludes that numbers of muons and tau leptons produced at an $e^{+} e^{-}$collider will be identical (up to systematic and statistical errors in analyzing the experimental data) if the new gauge symmetry, the $\mathrm{U}(1)^{\prime}$ symmetry under concern, exhibits identical $Z^{\prime}$ couplings for each fermion (at least lepton) family as happens in the standard electroweak theory.

In clear contrast to the left panel of Fig. 1, one observes that $\mu^{+} \mu^{-}$and $\tau^{+} \tau^{-}$differ by an order of magnitude if the $\mathrm{U}(1)^{\prime}$ symmetry possesses non-universal couplings to fermions (at least leptons). Indeed, $\sigma\left(e^{+} e^{-} \rightarrow \mu^{+} \mu^{-}\right)$is larger than $\sigma\left(e^{+} e^{-} \rightarrow \tau^{+} \tau^{-}\right)$by a factor of 6.5 , and this factor is related to $\mathrm{U}(1)^{\prime}$ charges listed in Table 1 and vector and axial-vector couplings in Table 3. Therefore, the right panel of Fig. 1 alone is sufficient for concluding that the number of $\mu^{+} \mu^{-}$and $\tau^{+} \tau^{-}$ events will significantly differ from each other if the new gauge symmetry, the U(1)' gauge symmetry under concern, exhibits different $Z^{\prime}$ couplings to different fermion (at least lepton) families.

Additionally we analyze the $\mathrm{U}(1)^{\prime}$ model at the Large Electron-Positron (LEP) collider which is closed at 2000 with $\sqrt{s}=209 \mathrm{GeV}$ and $140 \mathrm{pb}^{-1}$ luminosity. Fig. 2 is the production cross sections of muon and tau lepton final states with family non-universal $\mathrm{U}(1)^{\prime}$. It is clear in Fig. 2 that family non-universal $\mathrm{U}(1)^{\prime}$ signal is quite clean and distinguishable as the muon and tau lepton production cross sections are as much as several hundreds of picobarns. However, these productions are observed to be around few picobarns in various analysis $[5,6,14]$ and since such a clear and distinct signal has not been observed in LEP $[5,6]$, it can easily be said that family non-universal $Z^{\prime}$ lies beyond the discovery limit of LEP.

In conclusion, at linear colliders, which provide a perfect arena for precision measurements, one can determine if the new gauge symmetry, if any, which extends the SM gauge group exhibits family universal or non-universal couplings by simply counting the number of lepton pairs produced. This aspect 

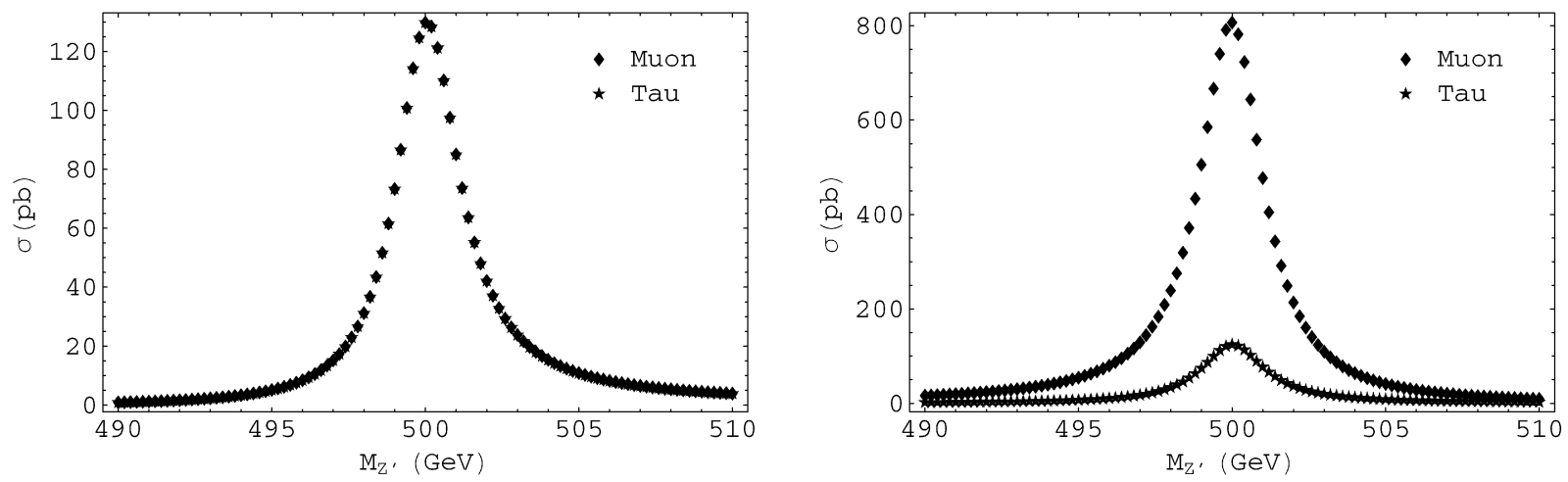

Fig. 1. The $\mu^{+} \mu^{-}$and $\tau^{+} \tau^{-}$productions at a future $e^{+} e^{-}$collider with $\sqrt{s}=500 \mathrm{GeV}$ for family universal $\mathrm{U}(1)^{\prime}$ (in the left panel) and family non-universal $\mathrm{U}(1)^{\prime}$ (in the right panel) models. The ratio between family non-universal and family universal cross sections varies with model parameters.

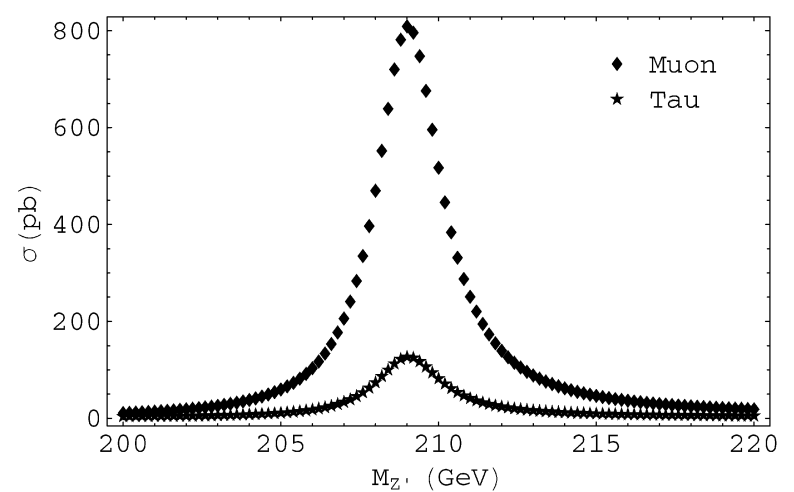

Fig. 2. Family non-universal $Z^{\prime}$ at LEP.

is quite important since family non-universality might signal anomaly cancellation in Abelian extended models as shown in [13].

\subsection{The hadron collider signatures}

The most important hadron machine to come up is the Large Hadron Collider (LHC) which is a proton-proton collider running at $\sqrt{s}=14 \mathrm{TeV}$ center of mass energy. At the parton level dilepton production processes are started by quark-anti-quark annihilation into lepton pairs via $s$-channel $\gamma, \mathrm{Z}$ and $\mathrm{Z}^{\prime}$ exchanges.

The hadronic cross section is related to the partonic one via

$$
\begin{aligned}
& \sigma\left(p p \rightarrow \ell^{+} \ell^{-}\right) \\
& \quad=\sum_{q, \bar{q}} C_{q \bar{q}} \int d x_{q} d x_{\bar{q}} \mathcal{P}_{q / A}\left(x_{q}\right) \mathcal{P}_{\bar{q} / B}\left(x_{\bar{q}}\right) \sigma\left(q \bar{q} \rightarrow \ell^{+} \ell^{-}\right),
\end{aligned}
$$

where $\mathcal{P}_{q / A}\left(x_{q}\right)$ stands for probability of finding parton (quark) $q$ within the hadron $A$ with a longitudinal momentum $x_{q}$ time that of the hadron. Moreover, $C_{q \bar{q}}$ stands for color averaging over initial-state partons and it equals $1 / 9$ for $q \bar{q}$ annihilation.

Depicted in Fig. 3 are $\sigma\left(p p \rightarrow e^{+} e^{-}\right)$and $\sigma\left(p p \rightarrow \mu^{+} \mu^{-}\right)$ for family universal (in the left panel) and non-universal (in the right panel) models. From the left panel it is clear that the two cross sections coincide, that is, an additional $\mathrm{U}(1)^{\prime}$ symmetry with universal couplings to fermion (at least lepton) families is expected to lead equal numbers of $e^{+} e^{-}$and $\mu^{+} \mu^{-}$pairs at the LHC. This observation is similar to what we found while analyzing ILC signatures in Section 3.1 above because of the fact that $\mathrm{U}(1)_{\eta}$ model possesses family universal couplings and mass difference between muon and electron cannot induce an observable effect on cross sections at such a high-energy collider $[5,6]$.

Similar to the right panel of Fig. 1, the right panel of Fig. 3 shows $e^{+} e^{-}$and $\mu^{+} \mu^{-}$production cross sections at the LHC with family non-universal $\mathrm{U}(1)^{\prime}$ model. The panel manifestly shows that $\sigma\left(p p \rightarrow e^{+} e^{-}\right)$is approximately 13 times smaller than $\sigma\left(p p \rightarrow \mu^{+} \mu^{-}\right)$because of unequal $\mathrm{U}(1)^{\prime}$ charges of electron and muon tabulated in Table 1 as well as their vector and axial-vector couplings given in Table 3 . Therefore, a family non-universal $\mathrm{U}(1)^{\prime}$, if any, can have observable signatures at the LHC via dilepton production processes.

We also examine the family non-universal $\mathrm{U}(1)^{\prime}$ model at $p-\bar{p}$ collisions with current bounds from Tevatron $(\sqrt{s}=$ $2 \mathrm{TeV}$ ). Fig. 4 shows muon and electron production cross sections at Tevatron with family non-universal U(1)' . Nevertheless the CDF [20-22] and D0 [23-25] experiments are expected to probe $\mathrm{Z}^{\prime}$ roughly in the range of $200-800 \mathrm{GeV}$ masses for various models, thus Tevatron experiments put strong limits on $\mathrm{Z}^{\prime}$ masses in agreement with the limits set by the LEP experiments. As it is understood in Fig. 4, family non-universal U(1) by being out of the limits is excluded at Tevatron with current bounds.

Before closing this section, we put strong emphasis on the fact that family non-universal $\mathrm{U}(1)^{\prime}$ offers observable signatures in dilepton signal in both linear and hadron colliders. In this sense, the LHC, which is expected to start operation in coming years, will be able to establish existence/absence of an additional $\mathrm{U}(1)^{\prime}$ symmetry in general and a family non-universal $\mathrm{U}(1)^{\prime}$ in particular. The latter will have easier observational characteristics because all that matters is the measurement of the ratios of events with different lepton flavors.

\section{Conclusion and outlook}

In this work we have contrasted family universal and nonuniversal $\mathrm{U}(1)^{\prime}$ models via their dilepton signatures in future linear (the ILC) and hadron (the LHC) colliders. These pro- 

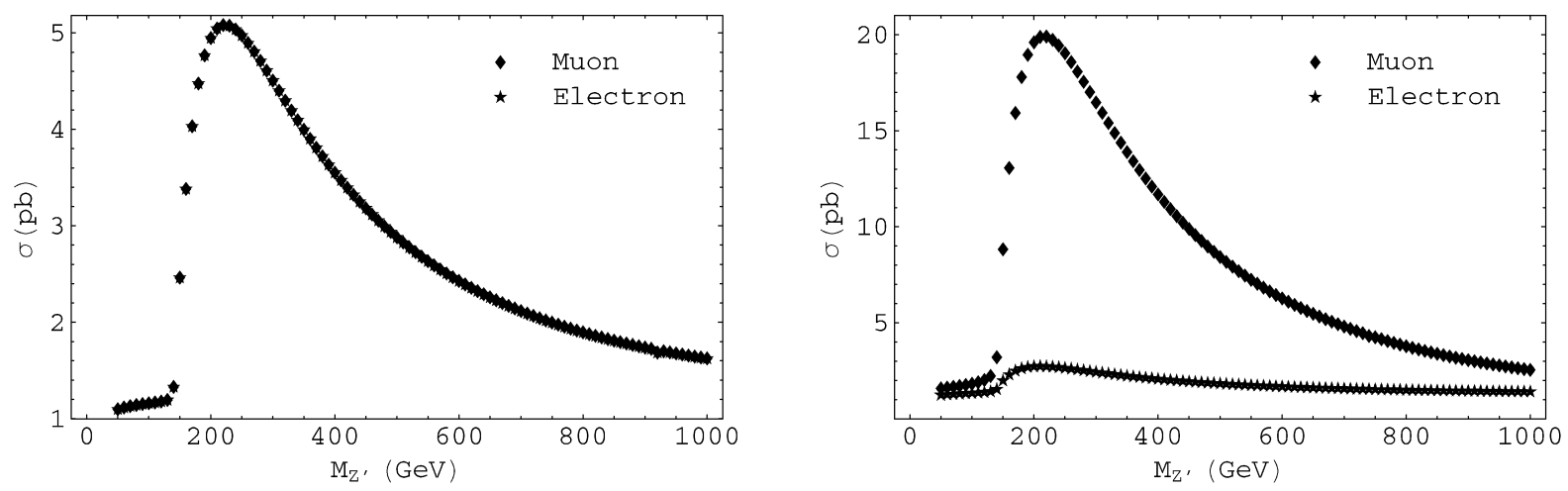

Fig. 3. The unpolarized $e^{+} e^{-}$and $\mu^{+} \mu^{-}$productions at the LHC for family universal (in the left panel) and non-universal (in the right panel) $\mathrm{U}(1)^{\prime}$ models. The ratio between family non-universal and family universal cross sections varies with model parameters.

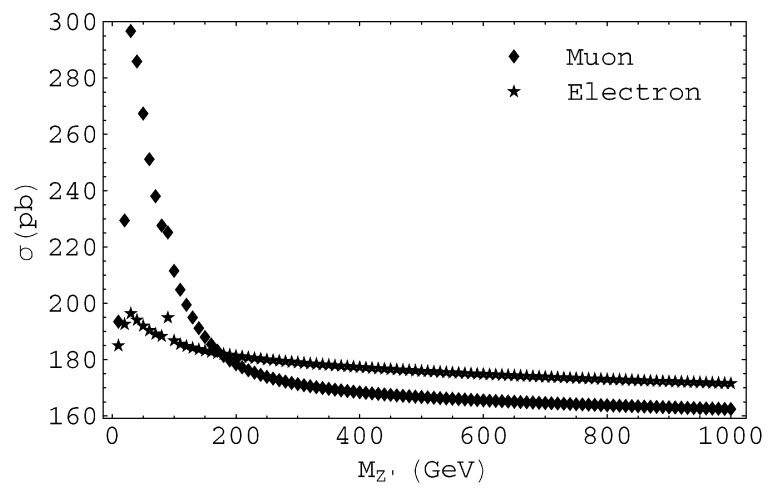

Fig. 4. Family non-universal $Z^{\prime}$ at Tevatron.

duction signatures are also observable in current colliders, and there are more stringent bounds on $Z^{\prime}$ from precision electroweak experiments and from direct searches in LEP $[5,6]$ and Tevatron [20-25]. The limits are model dependent because of the different couplings to fermions but typically the mass of a light $\mathrm{Z}^{\prime}$ is comparable with $\mathrm{Z}(\sim 200 \mathrm{GeV})$ and the heavy one is around $500-800 \mathrm{GeV}$ with small mixings [5-7,26].

Figs. 2 and 4 can be used in comparison between current and future colliders. Similar to ILC analysis Fig. 2 indicates a family non-universal $\mathrm{U}(1)^{\prime}$ model with current bounds in LEP and Fig. 4 is family non-universal $\mathrm{U}(1)^{\prime}$ at Tevatron in a similar fashion with LHC analysis. And again the family nonuniversality is at the difference in production cross sections of different flavors. As a result, family non-universal $Z^{\prime}$ is out of limits set by various experiments in LEP and Tevatron.

From discussions in Sections 3.1 and 3.2 we conclude that in both colliders (depending on systematic and statistical error bars in experimental data) one can establish existence/absence of a family non-universal $\mathrm{U}(1)^{\prime}$ model. This search is actually easier than direct $Z^{\prime}$ search since all that matters are the ratio of production cross sections of different lepton flavors.

For having a clearer sense of $Z^{\prime}$ search at colliders, it would be useful to analyze decay patterns of $Z^{\prime}$ boson into different flavors of matter. In general, a $Z^{\prime}$ boson of mass $M_{Z^{\prime}}$ decays into a fermion $f$ and anti-fermion $\bar{f}$ with a rate
$\Gamma_{\mathrm{Z}^{\prime} \rightarrow f \bar{f}}=M_{\mathrm{Z}^{\prime}}\left(\frac{g_{2}}{4 \cos \theta_{W}}\right)^{2}\left(\frac{v_{\mathrm{Z}^{\prime}}^{f^{2}}+a_{\mathrm{Z}^{\prime}}^{f^{2}}}{12 \pi}\right)$

directly proportional to $M_{Z^{\prime}}$. Therefore, if a certain number of $\mathrm{Z}^{\prime}$ bosons are produced ( $\mathrm{Z}^{\prime}$ bosons can be copiously produced at the LHC) then their decays into different fermion pairs give information about the underlying structure of the $\mathrm{U}(1)^{\prime}$ model.

Indeed, one expects at all grounds

$\frac{\Gamma_{\mathrm{Z}^{\prime} \rightarrow \mu+\mu-}}{\Gamma_{\mathrm{Z}^{\prime} \rightarrow \tau+\tau-}}=1 ; \quad \frac{\Gamma_{\mathrm{Z}^{\prime} \rightarrow \mu+\mu-}}{\Gamma_{\mathrm{Z}^{\prime} \rightarrow e+e-}}=1$

in any $\mathrm{U}(1)^{\prime}$ model (may it follow from $\mathrm{E}(6)$ or from strings) in which $Z^{\prime}$ couples to each lepton family in a universal fashion.

However, the same ratios of the decay rates become

$\frac{\Gamma_{\mathrm{Z}^{\prime} \rightarrow \mu+\mu-}}{\Gamma_{\mathrm{Z}^{\prime} \rightarrow \tau+\tau-}}=6.5 ; \quad \frac{\Gamma_{\mathrm{Z}^{\prime} \rightarrow \mu+\mu-}}{\Gamma_{\mathrm{Z}^{\prime} \rightarrow e+e-}}=13$

in the $\mathrm{U}(1)^{\prime}$ model of [13] in which $\mathrm{Z}^{\prime}$ couples to different lepton families differently (as listed in Tables 1 and 3). That the decay rates can significantly (depending on the model parameters) deviate from unity is a highly interesting signature for collider searches for a family non-universal $\mathrm{U}(1)^{\prime}$ gauge symmetry.

From the analyzes presented above we conclude that a $\mathrm{U}(1)^{\prime}$ gauge symmetry with non-universal couplings to lepton families offers unique observational signatures for collider searches via dilepton production.

\section{Acknowledgements}

The author thanks D.A. Demir for suggesting this project and several discussions and conversations while studying it. $\mathrm{He}$ is grateful to Kerem Cankoçak for his numerical help and guidance at various stages of this work. The author also thanks to participants of Ankara Winter Workshop, January 2007, for discussions and criticisms about this work. He gratefully acknowledges Turkish Academy of Sciences for financial support through the GEBIP grant (through D.A. Demir).

\section{References}

[1] J.E. Kim, H.P. Nilles, Phys. Lett. B 138 (1984) 150; 
D. Suematsu, Y. Yamagishi, Int. J. Mod. Phys. A 10 (1995) 4521, hepph/9411239;

M. Cvetič, P. Langacker, Mod. Phys. Lett. A 11 (1996) 1247, hep-ph/ 9602424;

V. Jain, R. Shrock, hep-ph/9507238;

Y. Nir, Phys. Lett. B 354 (1995) 107, hep-ph/9504312.

[2] J. Hewett, T. Rizzo, Phys. Rep. 183 (1989) 193.

[3] M. Cvetič, P. Langacker, Phys. Rev. D 54 (1996) 3570, hep-ph/9511378.

[4] C. Hill, E. Simmons, hep-ph/0203079.

[5] LEP Collaboration, hep-ex/0312023.

[6] ALEPH Collaboration, hep-ex/0511027.

[7] M. Cvetič, D.A. Demir, J.R. Espinosa, L.L. Everett, P. Langacker, Phys. Rev. D 56 (1997) 2861, hep-ph/9703317;

M. Cvetič, D.A. Demir, J.R. Espinosa, L.L. Everett, P. Langacker, Phys. Rev. D 58 (1998) 119905, Erratum.

[8] D.A. Demir, L.L. Everett, Phys. Rev. D 69 (2004) 015008, hep-ph/ 0306240.

[9] T. Han, P. Langacker, B. McElrath, hep-ph/0402064;

T. Han, P. Langacker, B. McElrath, hep-ph/0405244.

[10] D.A. Demir, N.K. Pak, Phys. Rev. D 57 (1998) 6609, hep-ph/9809357; H. Amini, New J. Phys. 5 (2003) 49, hep-ph/0210086.

[11] J. Kang, P. Langacker, T.j. Li, T. Liu, hep-ph/0402086.

[12] J. Erler, Nucl. Phys. B 586 (2000) 73, hep-ph/0006051.

[13] D.A. Demir, G.L. Kane, T.T. Wang, Phys. Rev. D 72 (2005) 015012, hep$\mathrm{ph} / 0503290$

[14] F. del Aguila, M. Cvetič, P. Langacker, Phys. Rev. D 48 (1993) R969, hep-ph/9303299;

F. del Aguila, M. Cvetič, P. Langacker, Phys. Rev. D 52 (1995) 37, hep$\mathrm{ph} / 9501390$;
F. del Aguila, M. Cvetič, Phys. Rev. D 50 (1994) 3158, hep-ph/9312329;

A. Leike, Phys. Lett. B 402 (1997) 374, hep-ph/9703263;

T. Appelquist, B.A. Dobrescu, A.R. Hopper, Phys. Rev. D 68 (2003) 035012, hep-ph/0212073;

M. Carena, A. Daleo, B.A. Dobrescu, T.M.P. Tait, Phys. Rev. D 70 (2004) 093009, hep-ph/0408098.

[15] J. Kang, P. Langacker, Phys. Rev. D 71 (2005) 035014, hep-ph/0412190.

[16] A. Fiandrino, P. Taxil, Phys. Rev. D 44 (1991) 3490;

P. Taxil, E. Tugcu, J.M. Virey, Eur. Phys. J. C 24 (2002) 149, hep-ph/ 0111242.

[17] J. Erler, P. Langacker, Phys. Rev. Lett. 84 (2000) 212, hep-ph/9910315; J. Erler, P. Langacker, hep-ph/0407097;

J. Erler, P. Langacker, Phys. Lett. B 456 (1999) 68, hep-ph/9903476;

P. Langacker, M.x. Luo, A.K. Mann, Rev. Mod. Phys. 64 (1992) 87;

U. Amaldi, et al., Phys. Rev. D 36 (1987) 1385.

[18] F. del Aguila, M. Quiros, F. Zwirner, Nucl. Phys. B 287 (1987) 419.

[19] K.S. Babu, C.F. Kolda, J. March-Russell, Phys. Rev. D 57 (1998) 6788, hep-ph/9710441.

[20] F. Abe, et al., CDF Collaboration, Phys. Rev. Lett. 79 (1997) 2192.

[21] F. Abe, et al., CDF Collaboration, Phys. Rev. D 51 (1995) 949.

[22] F. Abe, et al., CDF Collaboration, Phys. Rev. Lett. 68 (1992) 1463.

[23] S. Abachi, et al., D0 Collaboration, Phys. Lett. B 385 (1996) 471.

[24] V.M. Abazov, et al., D0 Collaboration, Phys. Rev. Lett. 87 (2001) 061802, hep-ex/0102048.

[25] B. Abbott, et al., D0 Collaboration, Phys. Rev. Lett. 82 (1999) 4769, hepex/9812010.

[26] P. Langacker, AIP Conf. Proc. 698 (2004) 1, hep-ph/0308145. 